\title{
Light backscattering polarization patterns from turbid media: theory and experiment
}

\author{
Milun J. Raković, George W. Kattawar, Mehrübe Mehrűbeoğlu, Brent D. Cameron, \\ Lihong V. Wang, Sohi Rastegar, and Gerard L. Coté
}

\begin{abstract}
We present both experimental measurements and Monte-Carlo-based simulations of the diffusely backscattered intensity patterns that arise from illuminating a turbid medium with a polarized laser beam. It is rigorously shown that, because of axial symmetry of the system, only seven elements of the effective backscattering Mueller matrix are independent. A new numerical method that allows simultaneous calculation of all 16 elements of the two-dimensional Mueller matrix is used. To validate our method we compared calculations to measurements from a turbid medium that consisted of polystyrene spheres of different sizes and concentrations in deionized water. The experimental and numerical results are in excellent agreement. (C) 1999 Optical Society of America

OCIS codes: $260.5430,290.1350,290.4210,290.7050$.
\end{abstract}

\section{Introduction}

A few recent studies have demonstrated that information on the properties of a turbid medium can be measured by shining a polarized laser beam onto a sample and then analyzing the state of polarization of the diffusely backscattered light. The investigated applications of this technique include the measurements of the average particle size, the scattering coefficients and the anisotropy factor of particle suspensions, ${ }^{1}$ cloud diagnostics, ${ }^{2,3}$ the study of biological materials, ${ }^{4-6}$ and the measurements of the average photon path length. ${ }^{7}$

To achieve full experimental characterization of the optical properties of the sample under investigation, Hielscher et al.6,8 used a Stokes vector/Mueller matrix approach to polarized light scattering. They generalized the concept of an effective Mueller matrix ${ }^{9}$ and measured the two-dimensional Mueller ma-

When this research was performed, all the authors were with Texas A\&M University, College Station, Texas 77843-4242. M. J. Raković and G. W. Kattawar are with the Department of Physics. M. Mehrúbeoğlu is with the Department of Electrical Engineering. B. D. Cameron, L. V. Wang, S. Rastegar, and G. L. Coté are with the Biomedical Engineering Program. M. J. Raković is on leave from the Institute of Physics, University of Belgrade, P.O. Box 57, Belgrade 11001, Yugoslavia. The e-mail address for G. W. Kattawar is kattawar@tamu.edu.

Received 23 June 1998; revised manuscript received 22 January 1999.

0003-6935/99/153399-10\$15.00/0

(C) 1999 Optical Society of America trix of backscattered light. In recent theoretical research, Ambirajan and Look ${ }^{10}$ used a Monte Carlo technique in studying the backscattering of a polarized laser beam from a plane-parallel medium. They studied the degree of polarization of the diffuse light when the incident beam was right circularly polarized. Raković and Kattawar ${ }^{11}$ theoretically analyzed the contribution to the light backscattering coming from double-scattering events and compared the results with the measurements of Pal and Carswell. ${ }^{2,3}$

In this paper we extend the research initiated in Ref. 12. We present a numerical method that allows simultaneous calculation of all 16 elements of the effective backscattering Mueller matrix. We prove that, because of axial symmetry of the system, there are only seven independent elements in this matrix and that the remaining elements are obtained from these by simple rotations or equality. To validate our method, we compare numerical simulations with measurements on polystyrene sphere suspensions. In Section 2 we define the effective backscattering Mueller matrix and represent it as a sum over all orders of multiple-scattered light. In Section 3, under rather general conditions, we derive important symmetry relations satisfied by the elements of the backscattering matrix. In Section 4 we develop a numerical Monte Carlo technique for calculation of the effective Mueller matrix. In Section 5 we compare theoretical results with experimental measurements. Finally, Section 6 contains concluding remarks. 


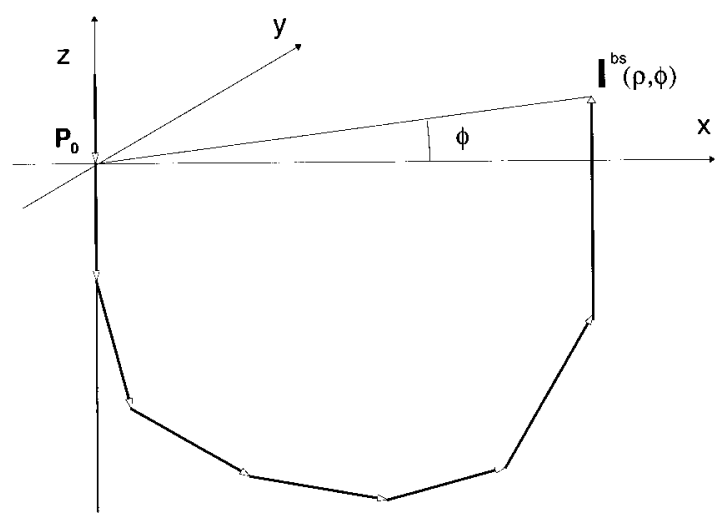

Fig. 1. Geometry of the multiple scattering.

\section{Backscattering Mueller Matrix}

We assume that the scattering of light is incoherent. The incoming narrow light beam propagates downward along the $z$ axis and is injected into an optically deep conservative scattering medium that is assumed to occupy the lower half-space, where $z \leq 0$ (see Fig. 1).

Let $\mathbf{F}_{0}$ be the Stokes vector corresponding to the irradiance of the incident laser beam with respect to the $x-z$ reference plane. We assume that the light crosses a small surface element $d s_{0}$, so that the vector describing the incident total light power is $\mathbf{P}_{0}=$ $\mathbf{F}_{0} d s_{0}$. After being scattered by particle scatterers, light leaves the medium in all possible directions. Let $\mathbf{I}^{\mathrm{bs}}(\rho, \phi)$ be the Stokes vector describing the radiance at the detector, i.e., at the point $(\rho, \phi, z=0)$ on the surface of the scattering medium, of the light that has been scattered exactly backward. For the reference plane we choose the plane parallel to the $x-z$ plane. Then

$$
\mathbf{I}^{\mathrm{bs}}(\rho, \phi)=\mu_{s}^{2} \mathbf{S}\left(\rho, \phi ; \mu_{s}, \mu_{T}\right) \mathbf{P}_{0},
$$

where $\mu_{s}$ and $\mu_{T}$ are scattering and extinction coefficients, respectively, ${ }^{13,14}$ and by definition $\mathbf{S}$ is the effective backscattering Mueller matrix. This matrix is obtained by solving a radiative transfer equation ${ }^{14}$ with the appropriate boundary conditions. In Section 5, we numerically calculate the matrix $\mathbf{S}$ using the Monte Carlo technique. Now we discuss the important general relations satisfied by the elements of $\mathbf{S}$.

If the scattering medium is homogeneous, the system possesses axial symmetry with respect to the $z$ axis. To see how this affects the form of the backscattering matrix, assume as above that the incoming light beam propagates downward along the $z$ axis and that the backscattered light leaves the medium at the point ( $\rho, \phi, z=0)$. However, let the plane of reference be the plane containing the incoming and backscattered light beam. Then

$$
\mathbf{I}^{\prime \text { bs }}(\rho, \phi)=\mu_{s}^{2} \mathbf{S}^{\prime}\left(\rho, \phi ; \mu_{s}, \mu_{T}\right) \mathbf{P}_{0}^{\prime},
$$

where primes correspond to a different reference plane. Now axial symmetry of the system is ex- pressed by the fact that the matrix $\mathbf{S}^{\prime}$ does not depend on $\phi$, i.e.,

$$
\mathbf{S}^{\prime}\left(\rho, \phi ; \mu_{s}, \mu_{T}\right) \equiv \tilde{\mathbf{S}}\left(\rho ; \mu_{s}, \mu_{T}\right),
$$

where $\tilde{\mathbf{S}}$ is the reduced backscattering matrix. From this it follows that the effective Mueller matrix takes the form

$$
\mathbf{S}\left(\rho, \phi ; \mu_{s}, \mu_{T}\right)=\mathbf{R}(-\phi) \tilde{\mathbf{S}}\left(\rho ; \mu_{s}, \mu_{T}\right) \mathbf{R}(-\phi),
$$

where $\mathbf{R}$ is the rotational Mueller matrix

$$
\mathbf{R}(\phi)=\left[\begin{array}{cccc}
1 & 0 & 0 & 0 \\
0 & \cos 2 \phi & \sin 2 \phi & 0 \\
0 & -\sin 2 \phi & \cos 2 \phi & 0 \\
0 & 0 & 0 & 1
\end{array}\right]
$$

that connects the two Stokes vectors that describe the same polarization state of the light beam but with respect to the two reference planes such that the first reference plane coincides with the second one after a counterclockwise rotation by the angle $\phi$ around the direction of light propagation.

From Eq. (4) we can see that the radial and azimuthal dependence of the backscattering matrix are factorized. Setting $\phi=0$ one obtains

$$
\tilde{\mathbf{S}}\left(\rho ; \mu_{s}, \mu_{T}\right)=\mathbf{S}\left(\rho, \phi=0 ; \mu_{s}, \mu_{T}\right) .
$$

Furthermore, the reduced backscattering matrix $\tilde{\mathbf{S}}$ can be represented as a sum over all orders of the multiple-scattered light, namely,

$$
\tilde{\mathbf{S}}\left(\rho ; \mu_{s}, \mu_{T}\right)=\sum_{n=2}^{\infty} \tilde{\mathbf{S}}_{n}\left(\rho ; \mu_{s}, \mu_{T}\right),
$$

where $\tilde{\mathbf{S}}_{n}$ denotes the contribution from the light that has been scattered exactly $n$ times by the scattering particles. $\tilde{\mathbf{S}}_{n}$ is itself a finite sum of terms $\tilde{\mathbf{S}}_{n}{ }^{i}$, where $0 \leq i \leq n-1$ is the number of times the light has been reflected from (and transmitted through) the surface (boundary) of the medium. Following the derivation by Raković and Kattawar, ${ }^{11}$ one can show that the (simplest) term $\tilde{\mathbf{S}}_{n}{ }^{0}$ takes the form (see Fig. 2)

$$
\begin{aligned}
\tilde{\mathbf{S}}_{n}{ }^{0}\left(\rho ; \mu_{s}, \mu_{T}\right)= & \mu_{s}{ }^{n-2} \int_{-\infty}^{0} \mathrm{~d} z_{1} \int_{z_{2} \leq 0} \mathrm{~d} \mathbf{r}_{2} \int_{z_{3} \leq 0} \mathrm{~d} \mathbf{r}_{3} \ldots \\
& \times \int_{z_{n-1} \leq 0} \mathrm{~d} \mathbf{r}_{n-1} \int_{-\infty}^{0} \mathrm{~d} z_{n} \exp \left(-\mu_{T}\left|z_{1}\right|\right) \\
& \times\left[\prod_{i=1}^{n-1} \frac{\exp \left(-\mu_{T}\left|\mathbf{r}_{i+1}-\mathbf{r}_{i}\right|\right)}{\left|\mathbf{r}_{i+1}-\mathbf{r}_{i}\right|^{2}}\right] \\
& \times \exp \left(-\mu_{T}\left|z_{n}\right|\right) \mathbf{R}\left(\phi_{n}\right) \mathbf{M}\left(\theta_{n}\right) \mathbf{R}\left(\phi_{n-1}\right) \ldots \\
& \times \mathbf{M}\left(\theta_{2}\right) \mathbf{R}\left(\phi_{12}\right) \mathbf{M}\left(\theta_{1}\right) \mathbf{R}\left(\phi_{1}\right),
\end{aligned}
$$

where $\mathbf{M}(\theta)$ is the Mueller matrix describing single scattering by particles. In the above, $\mathbf{r}_{i}(i=1, \ldots$, $n)$ are $n$ scattering points, $z_{i}<0$, and $\mathbf{r}_{1}=\left(x_{1}=0, y_{1}\right.$ $\left.=0, z_{1}<0\right)$ and $\mathbf{r}_{n}=\left(x_{n}=\rho, y_{n}=0, z_{n}<0\right)$; let also $\mathbf{r}_{0}=0$ and $\mathbf{r}_{n+1}=\left(x_{n+1}=\rho, y_{n}=0, z_{n}=0\right)$. $\theta_{i}$ are 


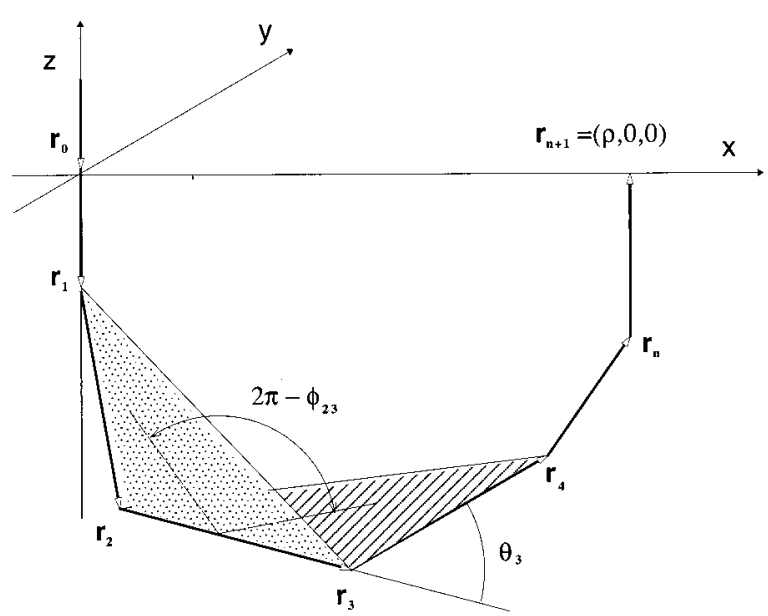

Fig. 2. Multiple-scattering trajectory: scattering points, scattering and rotational angles.

successive scattering angles (i.e., $\theta_{i}, 1<i<n$, is the angle between vectors $\mathbf{r}_{i+1}-\mathbf{r}_{i}$ and $\mathbf{r}_{i}-\mathbf{r}_{i-1} ; \theta_{1}$ is the angle between vectors $\mathbf{r}_{2}-\mathbf{r}_{1}$ and the negative $z$ axis; and $\theta_{n}$ is the angle between $\mathbf{r}_{n}-\mathbf{r}_{n-1}$ and the positive $z$ axis). $\phi_{i-1 i}$ are angles of rotation between successive scattering planes [i.e., $\phi_{i-1}, 2 \leq i \leq n$, is defined as an angle of rotation (in the positive direction) around a vector $\mathbf{r}_{i}-\mathbf{r}_{i-1}$ of the plane $\left(\mathbf{r}_{i-2}, \mathbf{r}_{i-1}, \mathbf{r}_{i}\right)$ which takes it to the plane $\left(\mathbf{r}_{i-1}, \mathbf{r}_{i}, \mathbf{r}_{i+1}\right) ; \phi_{1}$ is an angle of rotation around the negative $z$ axis of the $x-z$ plane which takes it to the plane $\left(\mathbf{r}_{0}, \mathbf{r}_{1}, \mathbf{r}_{2}\right)$; and $\phi_{n}$ is an angle of rotation around the positive $z$ axis of the plane $\left(\mathbf{r}_{n-1}, \mathbf{r}_{n}, \mathbf{r}_{n+1}\right)$ which takes it to the $x-z$ plane].

The terms $\tilde{\mathbf{S}}_{n}{ }^{l}$ with $0<i<n$ can be generated from Eq. (8) by inserting (in the matrix product) $i$ Fresnel matrices $\mathbf{T}$ that describe reflection from the surface of the medium. Recall that the Fresnel matrix can take two forms depending on the incident angle $\theta_{\text {in. }}$. If $\sin \theta_{\text {in }}>n_{12}=n_{2} / n_{1}$, where $n_{1}$ and $n_{2}$ are indices of refraction (medium 1 contains incident and reflected light and medium 2 contains transmitted light), then

$$
\mathbf{T}\left(\theta_{\text {in }}, n_{12}\right)=\left[\begin{array}{cccc}
1 & 0 & 0 & 0 \\
0 & 1 & 0 & 0 \\
0 & 0 & \cos \delta & -\sin \delta \\
0 & 0 & \sin \delta & \cos \delta
\end{array}\right],
$$

where $\delta=\delta\left(n_{12}, \cos \theta_{\text {in }}\right)$. If $\sin \theta_{\text {in }} \leq n_{12}$, then

$$
\mathbf{T}\left(\theta_{\mathrm{in}}, n_{12}\right)=\left[\begin{array}{cccc}
\frac{c_{1}{ }^{2}+c_{2}{ }^{2}}{2} & \frac{c_{1}{ }^{2}-c_{2}{ }^{2}}{2} & 0 & 0 \\
\frac{c_{1}{ }^{2}-c_{2}{ }^{2}}{2} & \frac{c_{1}{ }^{2}+c_{2}{ }^{2}}{2} & 0 & 0 \\
0 & 0 & c_{1} c_{2} & 0 \\
0 & 0 & 0 & c_{1} c_{2}
\end{array}\right]
$$

where $c_{i}=c_{i}\left(n_{12}, \cos \theta_{\text {in }}\right), i=1,2$.

It is convenient to use scaled (dimensionless) variables:

$$
\mathbf{r}_{s}=\mu_{T} \mathbf{r} .
$$

Introducing the above change of variables in Eq. (8), one obtains

$$
\begin{aligned}
\tilde{\mathbf{S}}_{n}{ }^{0}\left(\rho ; \mu_{s}, \mu_{T}\right)= & \bar{\omega}^{n-2} \mathbf{L}_{n}{ }^{0}\left(\rho_{s}\right) \\
= & \bar{\omega}^{n-2} \int_{-\infty}^{0} \mathrm{~d} z_{1} \int_{z_{2} \leq 0} \mathrm{~d} \mathbf{r}_{2} \int_{z_{3} \leq 0} \mathrm{~d} \mathbf{r}_{3} \ldots \\
& \times \int_{z_{n-1} \leq 0} \mathrm{~d} \mathbf{r}_{n-1} \int_{-\infty}^{0} \mathrm{~d} z_{n} \exp \left(-\left|z_{1}\right|\right) \\
& \times\left[\prod_{i=1}^{n-1} \frac{\exp \left(-\left|\mathbf{r}_{i+1}-\mathbf{r}_{i}\right|\right)}{\left|\mathbf{r}_{i+1}-\mathbf{r}_{i}\right|^{2}}\right] \\
& \times \exp \left(-\left|z_{n}\right|\right) \mathbf{R}\left(\phi_{n}\right) \mathbf{M}\left(\theta_{n}\right) \mathbf{R}\left(\phi_{n-1 n}\right) \ldots \\
& \times \mathbf{M}\left(\theta_{2}\right) \mathbf{R}\left(\phi_{12}\right) \mathbf{M}\left(\theta_{1}\right) \mathbf{R}\left(\phi_{1}\right),
\end{aligned}
$$

where $\rho_{s}=\mu_{T} \rho$ and $\bar{\omega}=\mu_{s} / \mu_{T}$ is the single-scattering albedo. The index $s$ is dropped in the integration variables and now $\mathbf{r}_{n}=\left(x_{n+1}=\rho_{s}, y_{n}=0, z_{n}<0\right)$. In scaled variables the expansion formula Eq. (7) becomes

$$
\tilde{\mathbf{S}}\left(\rho ; \mu_{s}, \mu_{T}\right)=\sum_{n=2}^{\infty} \bar{\omega}^{n-2} \mathbf{L}_{n}\left(\rho_{s}\right) .
$$

Again, the term $\mathbf{L}_{n}$ in the above sum describes the backscattered light that has been scattered $n$ times. For example, in the simplest and important case of the double-scattered light, $\mathbf{L}_{2}$ consists of two terms $\mathbf{L}_{2}$ $=\mathbf{L}_{2}{ }^{0}+\mathbf{L}_{2}{ }^{1}$, where $\mathbf{L}_{2}{ }^{0}$ corresponds to the light that is scattered twice before reaching the detector, whereas $\mathbf{L}_{2}{ }^{1}$ corresponds to the light that is additionally reflected from the surface of the medium before the second scattering. These two terms take relatively simple forms:

$$
\begin{aligned}
\mathbf{L}_{2}{ }^{0}\left(\rho_{s}\right)= & \int_{0}^{\infty} \int_{0}^{\infty} \frac{\mathrm{d} z_{1} \mathrm{~d} z_{2}}{r^{2}} \exp \left[-\left(z_{1}+z_{2}+r\right)\right] \mathbf{M}(\pi \\
& -\theta) \mathbf{M}(\theta), \\
r= & {\left[\rho_{s}{ }^{2}+\left(z_{1}-z_{2}\right)^{2}\right]^{1 / 2}, \tan \theta=\rho_{s} /\left(z_{2}-z_{1}\right), } \\
\mathbf{L}_{2}{ }^{1}\left(\rho_{s}\right)= & \int_{0}^{\infty} \int_{0}^{\infty} \frac{\mathrm{d} z_{1} \mathrm{~d} z_{2}}{r^{2}} \exp \left[-\left(z_{1}+z_{2}+r\right)\right] \mathbf{M}(\pi \\
& -\theta) T(\theta) \mathbf{M}(\pi-\theta), \\
r= & {\left[\rho_{s}{ }^{2}+\left(z_{1}+z_{2}\right)^{2}\right]^{1 / 2}, \tan \theta=\rho_{s} /\left(z_{1}+z_{2}\right) . }
\end{aligned}
$$

It can be shown that the above terms, in the case of the (Mie) scattering from the spherical particles, can be reduced to single integrals over the scattering angle $\theta$ and therefore easily calculated. ${ }^{11}$

\section{Symmetry Relations}

In this section we show that, under certain conditions, not all elements of the backscattering Mueller matrix, Eqs. (1) and (4), are independent. Consider the rather general case in which the scattering medium contains one kind of randomly distributed asymmetrical particles. Then the effective single- 
scattering Mueller matrix takes the form (Ref. 13, pp. 49 and 50)

$$
\mathbf{M}(\theta)=\left[\begin{array}{cccc}
M_{11} & M_{12} & M_{13} & M_{14} \\
M_{12} & M_{22} & M_{23} & M_{24} \\
-M_{13} & -M_{23} & M_{33} & M_{34} \\
M_{14} & M_{24} & -M_{34} & M_{44}
\end{array}\right] .
$$

Note also that the above form of the single-scattering Mueller matrix with ten independent elements also corresponds to optically active spheres (Ref. 15, p. 190). Now we define the projection matrix $\mathbf{P}$ :

$$
\mathbf{P}=\operatorname{diag}(1,1,-1,1), \mathbf{P}^{2}=\mathbf{E},
$$

where $\mathbf{E}$ is the identity matrix. Direct calculation shows that M, Eq. (14), satisfies the relation

$$
\mathbf{M}^{t}=\mathbf{P M P}
$$

where the superscript $t$ denotes transpose. [It can be shown that the matrix of Eq. (14) is in fact the most general matrix that satisfies the relation of Eq. (16).] In addition, from Eqs. (5), (9) and (10), it immediately follows that

$$
\mathbf{R}^{t}=\mathbf{P R P}, \mathbf{T}^{t}=\mathbf{P T P} .
$$

We now calculate the transpose of the matrix $\tilde{\mathbf{S}}_{n}{ }^{0}$ defined by Eq. (8).

Using Eqs. (16) and (17) one obtains

$$
\tilde{\mathbf{S}}_{n}{ }^{0 t}\left(\rho ; \mu_{s}, \mu_{T}\right)=\mathbf{P} \tilde{\mathbf{S}}_{n}{ }^{0}\left(\rho ; \mu_{s}, \mu_{T}\right) \mathbf{P}
$$

where

$$
\begin{aligned}
\tilde{\mathbf{S}}_{n}^{\prime}{ }^{0}\left(\rho ; \mu_{s}, \mu_{T}\right)= & \mu_{s}{ }^{n-2} \int_{-\infty}^{0} \mathrm{~d} z_{1} \int_{z_{2} \leq 0} \mathrm{~d} \mathbf{r}_{2} \int_{z_{3} \leq 0} \\
& \times \mathrm{d} \mathbf{r}_{3} \ldots \int_{z_{n-1} \leq 0} \mathrm{~d} \mathbf{r}_{n-1} \int_{-\infty}^{0} \mathrm{~d} z_{n} \\
& \times \exp \left(-\mu_{T}\left|z_{1}\right|\right) \\
& \times\left[\prod_{i=1}^{n-1} \frac{\left.\exp \left(-\mu_{T} \mid \mathbf{r}_{i+1}-\mathbf{r}_{i}\right)\right)}{\left|\mathbf{r}_{i+1}-\mathbf{r}_{i}\right|^{2}}\right] \\
& \times \exp \left(-\mu_{T}\left|z_{n}\right|\right) \mathbf{R}\left(\phi_{1}\right) \mathbf{M}\left(\theta_{1}\right) \mathbf{R}\left(\phi_{12}\right) \ldots \\
& \times \mathbf{M}\left(\theta_{n-1}\right) \mathbf{R}\left(\phi_{n-1}\right) \mathbf{M}\left(\theta_{n}\right) \mathbf{R}\left(\phi_{n}\right) . \quad(19
\end{aligned}
$$

We can see that $\tilde{\mathbf{S}}_{n}^{\prime}{ }^{0}$ differs from $\tilde{\mathbf{S}}_{n}{ }_{n}{ }^{0}$ only in the order of terms in the matrix product. In other words, the expression within the integral can be interpreted as the contribution of the light that is injected at the point $\mathbf{r}_{n+1}=\left(x_{n+1}=\rho, y_{n}=0, z_{n}=0\right)$ and scattered at the points $\mathbf{r}_{n}, \mathbf{r}_{n-1}, \ldots, \mathbf{r}_{1}$ before exiting the medium at the point $\mathbf{r}_{0}=0$. (This follows from the equality $\phi_{i-1 i}=\phi_{i-1}$.) Therefore it is also equal to the contribution of the light that enters the medium at $\mathbf{r}_{0}$, leaves at $\mathbf{r}_{n+1}$, and scatters at points $\mathbf{r}^{\prime}{ }_{1}, \mathbf{r}_{2}^{\prime}, \ldots, \mathbf{r}^{\prime}{ }_{n}$, where $\mathbf{r}^{\prime}{ }_{k}=\left(x_{k},-y_{k}, z_{k}\right)$, i.e., the trajectory $\mathbf{r}_{0}^{\prime}, \mathbf{r}_{1}^{\prime}, \ldots, \mathbf{r}_{n+1}^{\prime}$, is the mirror image with respect to the $x-z$ plane of the trajectory $\mathbf{r}_{0}$, $\mathbf{r}_{1}, \ldots, \mathbf{r}_{n+1}$. Thus $\tilde{\mathbf{S}}_{n}^{\prime}{ }^{0}$ is obtained from $\tilde{\mathbf{S}}_{n}{ }^{0}$ by transformation of the integration variables $y_{k} \rightarrow$ $-y_{k}$. Now, if the scattering medium is invariant under the transformation $y \rightarrow-y$ (which is obviously true for a homogeneous medium occupying the lower half-space), then the two integrals are equal, i.e., $\widetilde{\mathbf{S}}_{n}^{\prime}{ }^{0}=\widetilde{\mathbf{S}}_{n}{ }^{0}$, or by Eq. (18),

$$
\tilde{\mathbf{S}}_{n}{ }^{0 t}\left(\rho ; \mu_{s}, \mu_{T}\right)=\mathbf{P} \tilde{\mathbf{S}}_{n}{ }^{0}\left(\rho ; \mu_{s}, \mu_{T}\right) \mathbf{P} .
$$

The same relation is valid for $\tilde{\mathbf{S}}_{n}{ }^{i}$ because it differs from $\widetilde{\mathbf{S}}_{n}{ }^{0}$ only by the presence of Fresnel matrices $\mathbf{T}$ that also satisfy the necessary relation in Eq. (17). Therefore Eq. (20) holds for each $\tilde{\mathbf{S}}_{n}$ and consequently for their sum, i.e.,

$$
\tilde{\mathbf{S}}^{t}\left(\rho ; \mu_{s}, \mu_{T}\right)=\mathbf{P} \tilde{\mathbf{S}}\left(\rho ; \mu_{s}, \mu_{T}\right) \mathbf{P} .
$$

Equation (21) defines the most general form that the matrix $\tilde{\mathbf{S}}$ can take [see Eqs. (14) and (16)]:

$$
\tilde{\mathbf{S}}=\left[\begin{array}{cccc}
\tilde{S}_{11} & \tilde{S}_{12} & \tilde{S}_{13} & \tilde{S}_{14} \\
\tilde{S}_{12} & \tilde{S}_{22} & \tilde{S}_{23} & \tilde{S}_{24} \\
-\tilde{S}_{13} & -\tilde{S}_{23} & \tilde{S}_{33} & \tilde{S}_{34} \\
\tilde{S}_{14} & \tilde{S}_{24} & -\tilde{S}_{34} & \tilde{S}_{44}
\end{array}\right]
$$

Equations (4) and (22) now define the most general forms for the matrix elements of $\mathbf{S}$ which are

$$
\begin{aligned}
S_{11}(\rho, \phi)= & \tilde{S}_{11}(\rho), \\
S_{12}(\rho, \phi)= & \tilde{S}_{12}(\rho) \cos 2 \phi+\tilde{S}_{13}(\rho) \sin 2 \phi, \\
S_{13}(\rho, \phi)= & -\tilde{S}_{12}(\rho) \sin 2 \phi+\tilde{S}_{13}(\rho) \cos 2 \phi, \\
S_{14}(\rho, \phi)= & \tilde{S}_{14}(\rho), \quad S_{21}(\rho, \phi)=S_{12}(\rho, \phi), \\
S_{22}(\rho, \phi)= & \frac{\tilde{S}_{22}(\rho)-\tilde{S}_{33}(\rho)}{2}+\frac{\tilde{S}_{22}(\rho)+\tilde{S}_{33}(\rho)}{2} \cos 4 \phi \\
& +\tilde{S}_{23}(\rho) \sin 4 \phi, \\
S_{23}(\rho, \phi)= & \tilde{S}_{23}(\rho) \cos 4 \phi-\frac{\tilde{S}_{22}(\rho)+\tilde{S}_{33}(\rho)}{2} \sin 4 \phi, \\
S_{24}(\rho, \phi)= & \tilde{S}_{24}(\rho) \cos 2 \phi-\tilde{S}_{34}(\rho) \sin 2 \phi, \\
S_{31}(\rho, \phi)= & -S_{13}(\rho, \phi), \quad S_{32}(\rho, \phi)=-S_{23}(\rho, \phi), \\
S_{33}(\rho, \phi)= & -\frac{\tilde{S}_{22}(\rho)-\tilde{S}_{33}(\rho)}{2} \\
& +\frac{\tilde{S}_{22}(\rho)+\tilde{S}_{33}(\rho)}{2} \cos 4 \phi \\
& +\tilde{S}_{23}(\rho) \sin 4 \phi, \\
S_{34}(\rho, \phi)= & \tilde{S}_{24}(\rho) \sin 2 \phi+\tilde{S}_{34}(\rho) \cos 2 \phi, \\
S_{41}(\rho, \phi)= & S_{14}(\rho, \phi)=\tilde{S}_{14}(\rho), \\
S_{42}(\rho, \phi)= & S_{24}(\rho, \phi), S_{43}(\rho, \phi)=-S_{34}(\rho, \phi), \\
S_{44}(\rho, \phi)= & \tilde{S}_{44}(\rho) .
\end{aligned}
$$

From these general forms, it follows that there are only seven independent elements, $S_{11}(\rho), S_{12}(\rho, \phi)$, 
$S_{14}(\rho), S_{22}(\rho, \phi), S_{23}(\rho, \phi), S_{24}(\rho, \phi)$, and $S_{44}(\rho)$, and the other nine,

$$
\begin{aligned}
& S_{13}(\rho, \phi)=S_{12}(\rho, \phi+\pi / 4), \quad S_{21}(\rho, \phi)=S_{12}(\rho, \phi), \\
& S_{31}(\rho, \phi)=-S_{13}(\rho, \phi)=S_{12}(\rho, \phi-\pi / 4), \\
& S_{32}(\rho, \phi)=-S_{23}(\rho, \phi)=S_{23}(\rho, \phi \pm \pi / 4), \\
& S_{33}(\rho, \phi)=-S_{22}(\rho, \phi \pm \pi / 4), \\
& S_{34}(\rho, \phi)=S_{24}(\rho, \phi-\pi / 4), \\
& S_{41}(\rho, \phi)=S_{41}(\rho)=S_{14}(\rho), \quad S_{42}(\rho, \phi)=S_{24}(\rho, \phi), \\
& S_{43}(\rho, \phi)=-S_{34}(\rho, \phi)=S_{24}(\rho, \phi+\pi / 4),
\end{aligned}
$$

can be obtained by simple rotations at most.

The derived symmetry relations in Eqs. (24) [and the general form of the reduced backscattering matrix of Eq. (22)] hold, of course, if the assumptions made at the beginning of this section are satisfied, i.e., if the scattering medium contains one kind of randomly distributed asymmetrical particles, or optically active spheres. It can be shown that Eqs. (22) and (24) also hold when the medium that contains scattering particles is itself optically active because, in that case, the effective scattering matrix $\mathbf{S}$ contains additional rotational matrices of the form in Eq. (5), which describe rotations that are due to optical activity. Finally, for the symmetry relation to hold, it is not necessary that the scattering medium fill the entire half-space; it is sufficient for it to have rotational symmetry around the initial laser beam, e.g., it can be a cylinder of finite size whose axis coincides with the direction of the initial laser beam.

The reduced backscattering matrix, Eqs. (6) and (22), further simplifies in the simplest, and important, case of light scattering by homogeneous spheres. In that case, the single-scattering Mueller matrix was calculated by solving Maxwell's equations with appropriate boundary conditions (Mie theory), and it takes the relatively simple form ${ }^{13}$

$$
\mathbf{M}(\theta)=\left[\begin{array}{cccc}
a(\theta) & b(\theta) & 0 & 0 \\
b(\theta) & a(\theta) & 0 & 0 \\
0 & 0 & d(\theta) & -e(\theta) \\
0 & 0 & e(\theta) & d(\theta)
\end{array}\right],
$$

where the four independent elements, $a, b, d$, and $e$, expressed in terms of a series of Bessel functions, depend on the scattering angle $\theta$, the refractive index, and the size parameter of the scattering sphere.

Direct calculation shows that M, Eq. (25), satisfies, other than Eq. (16), the relation

$$
\mathbf{M}=\mathbf{Q} \mathbf{M Q},
$$

where $\mathbf{Q}$ is another projection matrix:

$$
\mathbf{Q}=\operatorname{diag}(1,1,-1,-1), \mathbf{Q}^{2}=\mathbf{E} .
$$

In addition, from Eqs. (5), (9), and (10), it immediately follows that

$$
\mathbf{R}^{t}=\mathbf{Q R Q}, \mathbf{T}^{t}=\mathbf{Q T Q} .
$$

With Eqs. (26)-(28), one can show [using a similar derivation that leads to Eq. (21)] that

$$
\tilde{\mathbf{S}}\left(\rho ; \mu_{s}, \mu_{T}\right)=\mathbf{Q} \tilde{\mathbf{S}}\left(\rho ; \mu_{s}, \mu_{T}\right) \mathbf{Q} .
$$

Therefore when the scatterers are homogeneous spheres, the reduced backscattering matrix satisfies both relations in Eq. (21) and Eq. (29), and the most general form it can take is

$$
\tilde{\mathbf{S}}=\left[\begin{array}{cccc}
\tilde{S}_{11} & \tilde{S}_{12} & 0 & 0 \\
\tilde{S}_{12} & \tilde{S}_{22} & 0 & 0 \\
0 & 0 & \tilde{S}_{33} & \tilde{S}_{34} \\
0 & 0 & -\tilde{S}_{34} & \tilde{S}_{44}
\end{array}\right] .
$$

In this case, the most general forms for the elements of the effective backscattering Mueller matrix $\mathbf{S}$, Eqs. (23), simplify by substituting $\tilde{S}_{13}(\rho)=\tilde{S}_{14}(\rho)=\widetilde{S}_{23}(\rho)$ $=\widetilde{S}_{24}(\rho)=\tilde{S}_{31}(\rho)=\tilde{S}_{32}(\rho)=\tilde{S}_{41}(\rho)=\tilde{S}_{42}(\rho)=0$. In this way the seven independent elements become

$S_{11}(\rho, \phi)=\tilde{S}_{11}(\rho), \quad S_{12}(\rho, \phi)=\tilde{S}_{12}(\rho) \cos 2 \phi$,

$S_{14}(\rho, \phi)=\widetilde{S}_{14}(\rho)=0$,

$S_{22}(\rho, \phi)=\frac{\tilde{S}_{22}(\rho)-\tilde{S}_{33}(\rho)}{2}+\frac{\tilde{S}_{22}(\rho)+\tilde{S}_{33}(\rho)}{2} \cos 4 \phi$,

$S_{23}(\rho, \phi)=-\frac{\tilde{S}_{22}(\rho)+\tilde{S}_{33}(\rho)}{2} \sin 4 \phi$,

$S_{24}(\rho, \phi)=-\tilde{S}_{34}(\rho) \sin 2 \phi, \quad S_{44}(\rho, \phi)=\tilde{S}_{44}(\rho)$,

whereas the other nine elements are obtained through symmetry relations, Eqs. (24), or explicitly

$$
\begin{aligned}
S_{13}(\rho, \phi) & =S_{12}(\rho, \phi+\pi / 4)=-\tilde{S}_{12}(\rho) \sin 2 \phi, \\
S_{21}(\rho, \phi) & =S_{12}(\rho, \phi), \\
S_{31}(\rho, \phi) & =-S_{13}(\rho, \phi), \quad S_{32}(\rho, \phi)=-S_{23}(\rho, \phi), \\
S_{33}(\rho, \phi) & =-S_{22}(\rho, \phi \pm \pi / 4) \\
& =-\frac{\tilde{S}_{22}(\rho)-\tilde{S}_{33}(\rho)}{2}+\frac{\tilde{S}_{22}(\rho)+\tilde{S}_{33}(\rho)}{2} \cos 4 \phi, \\
S_{34}(\rho, \phi) & =S_{24}(\rho, \phi-\pi / 4)=\tilde{S}_{34}(\rho) \cos 2 \phi, \\
S_{41}(\rho, \phi) & =S_{14}(\rho, \phi)=0, \\
S_{42}(\rho, \phi) & =S_{24}(\rho, \phi), \quad S_{43}(\rho, \phi)=-S_{34}(\rho, \phi) . \quad(30 \mathrm{~b})
\end{aligned}
$$

From Eqs. (30a) and (30b) we can see that, in the case of spherical scatterers, all matrix elements are defined by six functions, $\tilde{S}_{11}(\rho), \tilde{S}_{12}(\rho), \tilde{S}_{22}(\rho), \tilde{S}_{33}(\rho)$, $\tilde{S}_{34}(\rho)$, and $\tilde{S}_{44}(\rho)$, that depend only on radial distance. We note at the end of this section that the symmetry relations similar to those in Eqs. (21) and (29) are, in a different context, discussed in Ref. 16.

\section{Monte Carlo Calculations}

We used the Monte Carlo method to calculate the effective backscattering Mueller matrix for the case of spherical scatterers. Essentially, we represent $\mathbf{S}$, Eqs. (4), (7), and (8), as the sum over a large number of randomly chosen photon trajectories that all start at the origin $\mathbf{r}_{0}=0$. Each trajectory is defined by the collection of points $\mathbf{r}_{i}, i=1,2, \ldots, n$, where the 
light scatterings take place and there are possible additional points of reflection from the boundary of the medium.

The trajectories are generated in the following way (we describe only the trajectories without boundary reflection; the generalization is straightforward). Because the light enters the medium propagating downward along the $z$ axis, the first scattering point $\mathbf{r}_{1}=\left(0,0, z_{1}<0\right)$ is determined by choosing the length $l_{1}=\left|z_{1}\right|$. This, as well as all subsequent free path lengths $l_{i+1}=\left|\mathbf{r}_{i+1}-\mathbf{r}_{i}\right|, i=1,2, \ldots$, are chosen using a random number generator so their probability distribution is $w(l)=\mu_{T} \exp \left(-\mu_{T} l\right)$. Suppose now that the points $\mathbf{r}_{i}, i=1,2, \ldots, k$ and $l_{k+1}$ are already defined, then the next point $\mathbf{r}_{k+1}$ is determined by choosing the unit vector $\left|\mathbf{r}_{k+1}-\mathbf{r}_{k}\right| /$ $l_{k+1}$. This is defined by the scattering angle $\theta_{k}$ and the rotational angle $\phi_{k-1 k}$ that are defined in Section 2. Azimuth angles $\phi$ are chosen according to the uniform distribution $w(\phi)=1 / 2 \pi$, whereas the scattering angles $\phi$ are chosen according to the distribution defined by an element of the single-scattering matrix $w(\theta)=2 \pi a(\theta) \sin \theta$ [see Eq. (25)].

To each point $\mathbf{r}_{i}$, we associate a matrix $\mathbf{M}_{i}$ and weight $w_{i}$ using the following procedure. To $\mathbf{r}_{0}$ we associate $\mathbf{M}_{0}=1$ and $w_{0}=1$. Suppose that $\mathbf{M}_{i}$ and $w_{i}(i=1,2, \ldots, k)$ are already defined, then $\mathbf{M}_{k+1}=$ $\mathbf{M}\left(\theta_{k}\right) \mathbf{R}\left(\phi_{k}\right) \mathbf{M}_{k} / a\left(\theta_{k}\right)$ and $w_{k+1}=\bar{\omega} w_{k}$. If between $\mathbf{r}_{k}$ and $\mathbf{r}_{k+1}$ there is a point of reflection from the surface (boundary) of the medium, then the matrix $\mathbf{M}_{k+1}$ is additionally multiplied by the appropriate Fresnel matrix. The trajectory is not infinite; it is truncated when the product $\left(\mathbf{M}_{k}\right)_{11} w_{k}$ is below some predetermined cutoff value. (Let us mention that in the Monte Carlo simulations presented in Section 6, the average number of collisions per trajectory was ten.)

Each triplet $\mathbf{r}_{i}, \mathbf{M}_{i}, w_{i}$ gives a contribution to the backscattering matrix $\mathbf{S}$ in the following way. Effectively, the resulting matrix $\mathbf{S}$ is given as a matrix function defined on a discrete set of points that define a certain grid on the $z=0$ plane. Now, the $i$ th triplet gives an additive contribution to the value of $\mathbf{S}$ associated with the grid point closest to the point $x_{i}$, $y_{i}$, with the contribution being

$$
w_{i} \exp \left(-\mu_{T}\left|z_{i}\right|\right) \mathbf{R}\left(\phi_{i}{ }^{\prime}\right) \mathbf{M}\left(\theta_{i}{ }^{\prime}\right) \mathbf{M}_{i},
$$

where $\theta_{i}{ }^{\prime}$ is the angle between $\mathbf{r}_{i}-\mathbf{r}_{i-1}$ and the positive $z$ axis, whereas $\phi_{i}{ }^{\prime}$ is the angle between the plane $\left(\mathbf{r}_{i-1}, \mathbf{r}_{i}, \mathbf{r}_{i}^{\prime}\right)$ and the $x-z$ plane, where $\mathbf{r}_{i}^{\prime}=\left(x_{i}\right.$, $\left.y_{i}, 0\right)$. We can see that each trajectory effectively carries contributions of as many trajectories as there are scattering points along the trajectory.

The procedure described above uses the Monte Carlo method to calculate the matrix $\mathbf{S}$, although we have seen that, due to Eq. (4), it is sufficient to calculate the matrix $\tilde{\mathbf{S}}$. From Eq. (4) it follows that, to calculate this matrix, it is sufficient to sandwich each contribution [expression (31)] between the two matrices $\mathbf{R}\left(\phi_{i}\right)$ and to associate the result to the point $\rho_{i}$, where $\left(\rho_{i}, \phi_{i}, z_{i}\right)$ are the cylindrical coordinates of $\mathbf{r}_{i}$. We note that this last transformation improves our computational statistics enormously.

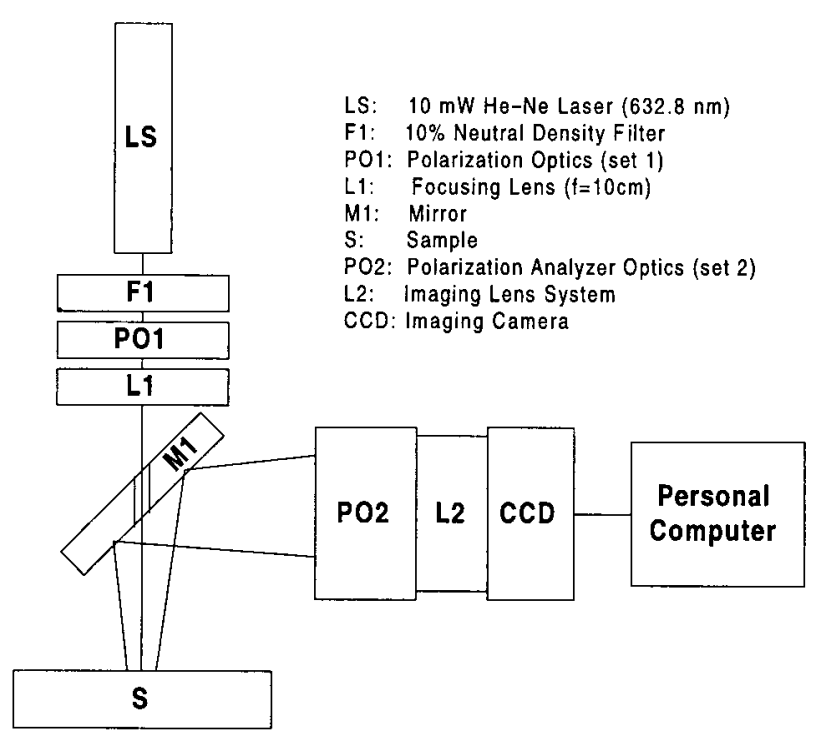

Fig. 3. Schematic diagram of the experimental setup.

\section{Comparison with Experiment}

A schematic view of the experimental apparatus used for collection of the diffuse backscattered images is shown in Fig. 3. A collimated light beam emitted by a $10-\mathrm{mW} \mathrm{He}-\mathrm{Ne}$ laser at a wavelength of $632.8 \mathrm{~nm}$ passes through a $10 \%$ transmittance neutral density filter (FI). This beam is then polarized by various polarization optics (PO1) (i.e., linear and circular polarizers) to obtain the desired input polarization. This polarized beam is then focused with the lens (L1, $f=10 \mathrm{~cm})$ through a hole $(d \approx 2 \mathrm{~mm})$ in a mirror (M1), mounted at $45 \mathrm{deg}$, onto the sample. The diffusely backscattered light from the sample is transmitted through a polarization analyzer (PO2) and imaged using a lens (L2) onto a cooled 12-bit CCD camera (SpectraSource, Westlake Village, Calif.). The polarization analyzer consisted of a variety of optics that were interchanged to analyze a specific type of polarized light (i.e., vertical, horizontal, $\pm 45^{\circ}$ linear, and left and right circularly polarized light), and these images were used to reconstruct the Mueller matrix. The CCD array size is $336 \times 243$ pixels with a pixel size of $10 \mu \mathrm{m} \times 10 \mu \mathrm{m}$. Each image was collected using an exposure time of $1.7 \mathrm{~s}$.

The phantom used in the experiment was comprised of a suspension of polystyrene spheres of 2.02- $\mu \mathrm{m}$ diameter (Ernest F. Fullam, Latham, N.Y.). The sample was created by diluting $0.075 \mathrm{ml}$ of a 10 -wt\% suspension of polystyrene spheres with $15 \mathrm{ml}$ of deionized water. The index of refraction of the spheres is 1.59, and the sample has a relative index of refraction $n / n_{w}$ of 1.192 where $n_{w}$ is the index of refraction for water. The sample has a scattering coefficient $\left(\mu_{s}\right)$ and anisotropy factor $(g)$ of $11.88 \mathrm{~cm}^{-1}$ and 0.912 , respectively, at $632.8 \mathrm{~nm}$, and has a negligible absorption coefficient $\mu \approx 0(\bar{\omega} \approx 1)$. Finally, the transport mean free path is ${ }^{4} \mathrm{mfp}^{\prime}=1 /\left[\mu_{a}+\right.$ $\left.\mu_{s}(1-g)\right]=0.957 \mathrm{~cm}$.

Figure 4 shows, side by side, elements of the effec- 


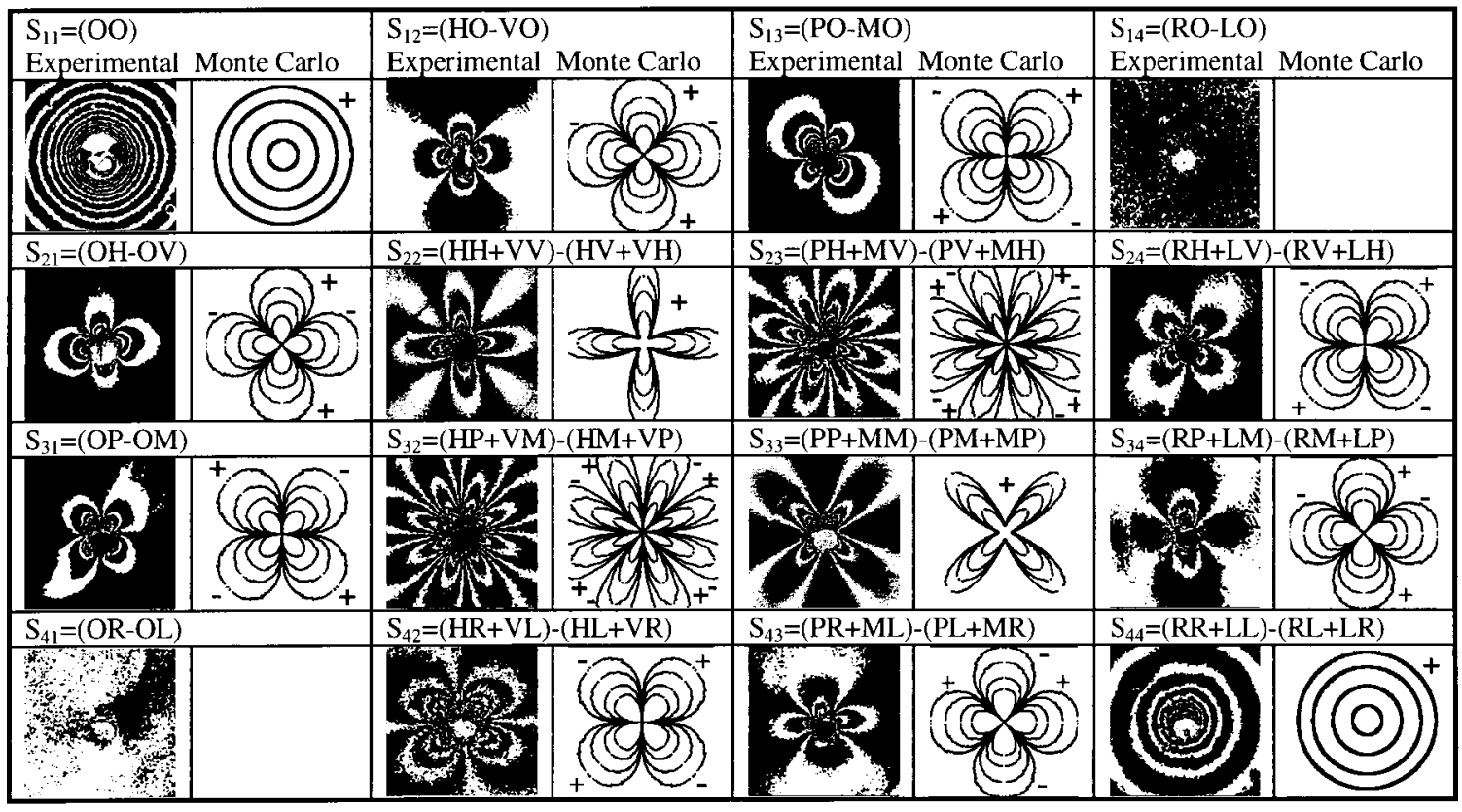

Fig. 4. Experimental and Monte Carlo backscattering Mueller matrix. The phantom was comprised of a 0.05 -wt\% suspension of polystyrene spheres (of diameter $2.02 \mu \mathrm{m}$ ) in deionized water. The approximate size of each image is $1.6 \mathrm{~cm} \times 1.6 \mathrm{~cm}$. Light wavelength was $632.8 \mathrm{~nm}$. The normalized (see the text) absolute values corresponding to the contours of the theoretical matrix elements are $S_{11}$ : $266,111,69,49 \times 10^{-4} ; S_{12}, S_{21}, S_{13}, S_{31}: \quad 15,6,2.5,1 \times 10^{-4} ; S_{22}, S_{33}: \quad 38,20,14,10 \times 10^{-4} ; S_{23}, S_{32}: \quad 26,15,10,6 \times 10^{-4} ; S_{24}$, $S_{34}, S_{42}, S_{43}: 10,5,2,1 \times 10^{-4} ; S_{44}: 129,60,38,28 \times 10^{-4}$. The smaller values correspond to the contours located farther from the center of each plot.

tive backscattering Mueller matrix obtained both experimentally and theoretically. The approximate size of each image is $1.6 \mathrm{~cm} \times 1.6 \mathrm{~cm}$. (It appears that, for distances that exceed two transport mean free paths approximately equal to $1.9 \mathrm{~cm}$, the azimuthal dependence of the patterns becomes less pronounced because multiple scatterings tend to randomize the polarization state of the light.)

The results of the Monte Carlo calculations are given in the form of the contour plots; each matrix element is represented by several contours (i.e., sets in the $x-y$ plane corresponding to certain fixed values of the corresponding element). Using the method of Section 4, we first obtained the reduced matrix $\tilde{\mathbf{S}}$, Eq. (30), or equivalently, six functions: $\tilde{S}_{11}(\rho), \tilde{S}_{12}(\rho)$, $\tilde{S}_{22}(\rho), \tilde{S}_{33}(\rho), \tilde{S}_{34}(\rho)$, and $\tilde{S}_{44}(\rho)$. Then from Eqs. (30a) and (30b) we can calculate any contour corresponding to each element of the matrix $\mathbf{S}$. When labeling the contours, shown in Fig. 4, by the numerical values (of the corresponding matrix elements), we adopted the normalization that the center in the contour plot of the matrix element $S_{11}$ corresponds to unity. Let us emphasize at this point that many important features of the plots shown in Fig. 4 can be predicted from the azimuthal dependence of the matrix elements that is given in Eqs. (30a) and (30b). For example, the formula for the matrix element $S_{12}$, Eqs. (30a), shows that for any given contour there is another one of the same shape but rotated by $90 \mathrm{deg}$ and that corresponds to the same absolute value but the opposite sign of the matrix element. Indeed in Fig. 4 we can see that to $S_{12}$ correspond pairs of contours in the shape of horizontal and vertical number eight that have exactly opposite values of the matrix element. From Eqs. (30a) and (30b) one can easily deduce similar symmetry properties for each of the elements in Fig. 4.

To determine each of the 16 experimental matrix elements, a total of 49 images are taken at various combinations of input and output analyzer polarization states. Each of the 16 experimental elements is calculated by adding or subtracting a series of images. The individual images are represented by a two-letter combination that denotes the input polarization and output analyzer orientation (i.e., HV denotes horizontal input polarized light and a vertical polarization analyzer). The corresponding symbols denoting polarization are $\mathrm{V}$, vertical; $\mathrm{H}$, horizontal; $\mathrm{P}$, $+45^{\circ}$; M, $-45^{\circ}$; R, right; L, left; and $\mathrm{O}$, open or no polarization optics. Note that to obtain correct images one also has to take care of the effect of the mirror M1. Namely, the experimental setup in Fig. 3 directly measures a matrix $\mathbf{S}^{\exp }=\mathbf{S M} 1$ where $\mathbf{S}$ is the desired backscattering matrix, whereas $\mathbf{M} 1$ is the Mueller matrix of the realistic mirror M1. In our experiment we used an aluminum mirror with the Mueller matrix (for an incident angle of $45 \mathrm{deg}$ ):

$$
\mathbf{M} 1=\left[\begin{array}{cccc}
0.82 & 0.055 & 0 & 0 \\
0.055 & 0.82 & 0 & 0 \\
0 & 0 & -0.79 & -0.20 \\
0 & 0 & 0.20 & -0.79
\end{array}\right] .
$$


Therefore after the matrix $\mathbf{S}^{\exp }$ is determined as explained above, it has to be multiplied from the right by $\mathbf{M} 1^{-1}$.

In Fig. 4 the experimental matrix elements are represented by several black and white regions, each region corresponding to a certain range of values of the matrix element. (Fluctuation of the experimental results, see below, did not allow for precise determination of a single contour.) Boundaries between black and white regions then approximately define the contours, which have the same shape as those obtained by numerical calculations, and in both cases the contours located farther from the center correspond to smaller absolute values of the matrix elements. Figure 4 also shows that the experiment did not produce any pattern for the matrix elements $S_{14}$ and $S_{41}$; only a weak signal was detected. On the other hand, theory predicts that these elements vanish for spherical scatterers [see Eqs. (30a) and (30b)]. Accordingly, our numerical calculations produced only negligible fluctuations around zero for the elements $\widetilde{S}_{14}$ and $\widetilde{S}_{41}$.

The results shown in Fig. 4 demonstrate good qualitative agreement between theory and experiment for the intensity patterns of the two-dimensional backscattered Mueller matrix elements in the case of multiple scattering of the incident radiation from a suspension of polystyrene spheres. However, we now make a more direct and quantitative comparison between theory and experiment by presenting the results describing cross-polarized backscattering patterns for various concentrations of spherical scatterers and different particle sizes. The directly measured cross-polarized patterns ${ }^{3,7,11}$ correspond to the HV orientation of the experimental setup, i.e., horizontal (along the $x$-axis) polarization of the incident light and vertical (along the $y$-axis) polarization analyzer. In terms of the Mueller matrix elements, the corresponding intensity patterns are (up to a multiplicative factor)

$$
C(\rho, \phi)=S_{11}(\rho, \phi)+S_{12}(\rho, \phi)-S_{21}(\rho, \phi)-S_{22}(\rho, \phi) .
$$

From Eqs. (23) and (32) it follows that

$$
\begin{aligned}
C(\rho, \phi)= & \tilde{S}_{11}(\rho)-\frac{\tilde{S}_{22}(\rho)-\tilde{S}_{33}(\rho)}{2}-\frac{\tilde{S}_{22}(\rho)+\tilde{S}_{33}(\rho)}{2} \\
& \times \cos 4 \phi-\tilde{S}_{23}(\rho) \sin 4 \phi,
\end{aligned}
$$

and in the case of spherical scatterers, Eq. (30), one obtains

$$
\begin{aligned}
C(\rho, \phi)= & \tilde{S}_{11}(\rho)-\frac{\tilde{S}_{22}(\rho)-\tilde{S}_{33}(\rho)}{2} \\
& -\frac{\tilde{S}_{22}(\rho)+\tilde{S}_{33}(\rho)}{2} \cos 4 \phi .
\end{aligned}
$$

We can see that our theory predicts $(\cos 4 \phi)$ azimuthal dependence in accordance with cloverlike patterns that were observed experimentally. 3,7

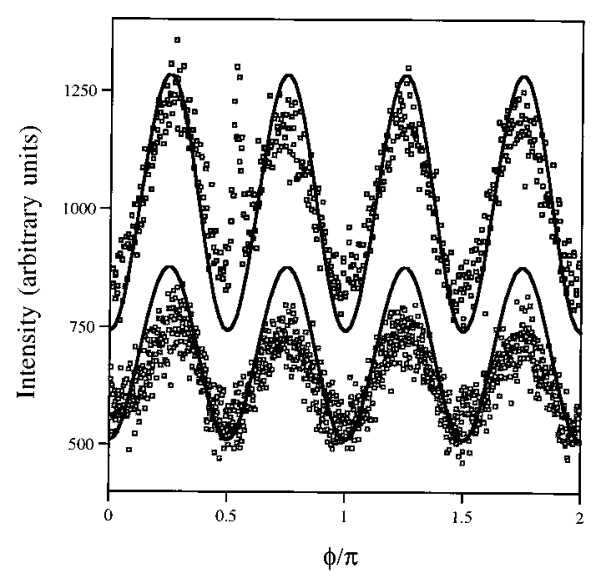

Fig. 5. Experimental (scattered symbols) and Monte Carlo (solid curves) results for the azimuthal dependence of crossed patterns $C(\rho, \phi)$, Eq. (33), for polystyrene spheres of diameter $2.02 \mu \mathrm{m}$ and a concentration of $0.05 \%$. The upper data correspond to $2-\mathrm{mm}$ radial distance, whereas the lower data correspond to $4 \mathrm{~mm}$. Light wavelength was $632.8 \mathrm{~nm}$.

In Figs. 5 and 6 we show experimental and Monte Carlo results for the azimuthal dependence of crossed patterns for various radial distances and particle sizes. In Fig. 5 we used the same suspension as in Fig. 4, whereas in Fig. 6 we used water suspensions of $0.482-\mu \mathrm{m}$ polystyrene spheres for three different concentrations. Figures 5 and 6 demonstrate excellent quantitative agreement between theory and experiment: Theoretical curves appear to be almost exact envelopes of the fluctuating experimental results.

Finally in Fig. 7 we compared experimental and Monte Carlo images of the backscattering crossed polarization patterns that correspond to the suspension used in Fig. 6 . Inner and outer patterns in each image are the theoretical results, whereas the middle pattern in each image is the experimental result. We can see that the expected cloverlike patterns s $^{3,7}$ are obtained. However, Fig. 7 shows that, as the concentration of the scatterers increases, the patterns are located in a smaller vicinity of the point of incidence of the laser beam. We note that, although the patterns are obtained for perfect homogeneous spheres, Eq. (33) predicts that such patterns should emerge, possibly slightly rotated, for any kind of identical particles in random orientation, provided that the concentration is sufficiently low.

\section{Conclusion}

In this research we have demonstrated that the theory of incoherent light scattering by particles is a satisfactory framework for the description for light backscattering from turbid media. Our theory predicted, and our experiment confirmed, that the effective backscattering Mueller matrix possesses important symmetry relations: Only seven matrix elements are independent and the rest can be obtained by simple rotations. These symmetry relations hold under general conditions: The scattering 

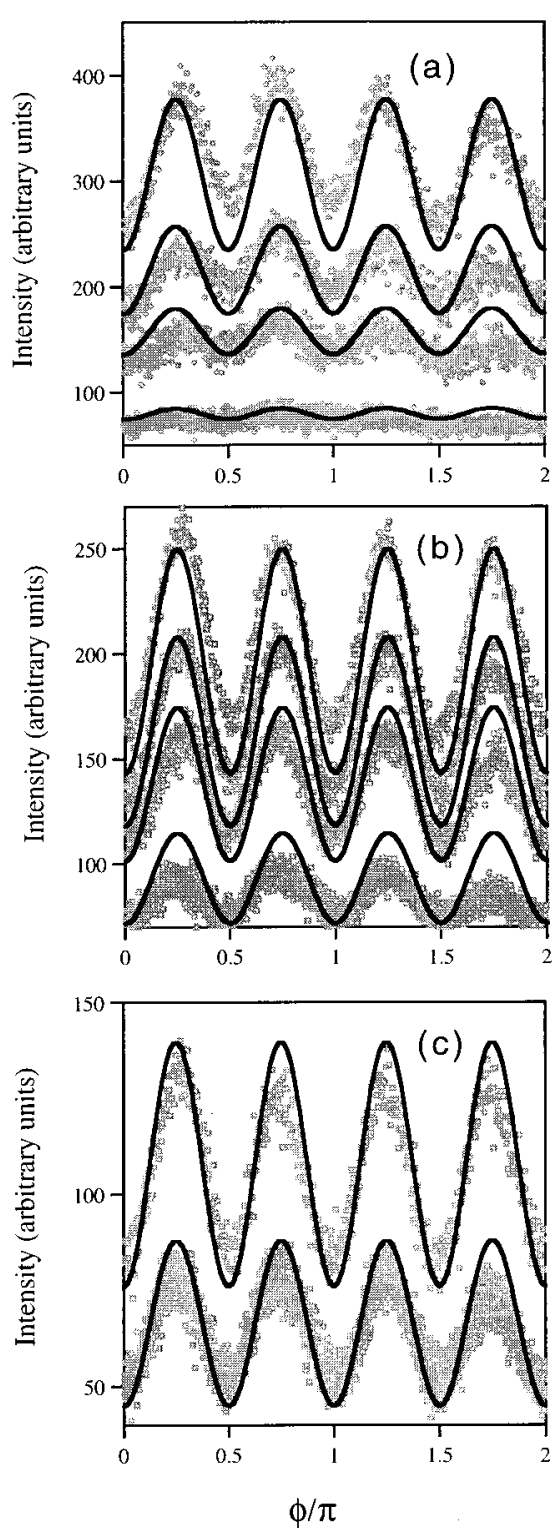

Fig. 6. Experimental (scattered symbols) and Monte Carlo (solid curves) results for the azimuthal dependence of crossed patterns $C(\rho, \phi)$, Eq. (33), for polystyrene spheres of diameter $0.482 \mu \mathrm{m}$ and three different concentrations: (a) $0.1 \%$, (b) $0.05 \%$, and (c) $0.025 \%$. In (a)-(c), data at the top of the plots correspond to $2-\mathrm{mm}$ radial distance, whereas data on the bottom of the plots correspond to $4 \mathrm{~mm}$. In (a) and (b), the other two data correspond to radial distances of 3 and $4 \mathrm{~mm}$. Light wavelength was $632.8 \mathrm{~nm}$.

medium should be invariant under rotations around the initial laser beam direction and should contain an ensemble of identical (possibly asymmetric) scattering particles in random orientations. The second condition can be weaker, in a sense that the scatterers may constitute a finite number of different ensembles of (identical) particles. In other words, the symmetry relations will break down only if scattering particles are all virtually different and cannot be classified in several ensembles of identical particles.

The laser beam in the experiment was coherent, and nevertheless the light scattering treated in the
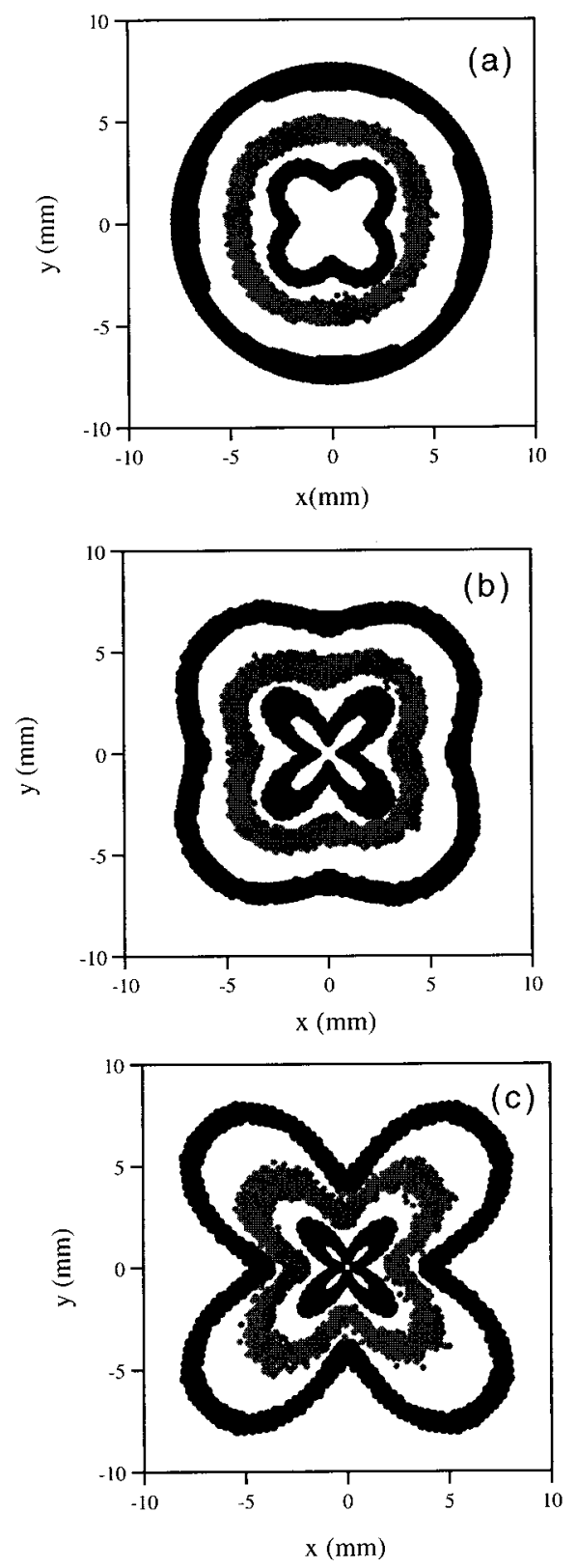

Fig. 7. Experimental and theoretical (Monte Carlo) backscattering crossed-polarization patterns for the suspension from Fig. 6 and three concentrations: (a) $0.1 \%$, (b) $0.05 \%$, and (c) $0.025 \%$. Inner and outer patterns in each image are the theoretical results, whereas the middle pattern in each image is the experimental result.

theoretical simulations was incoherent. This is justified by the fact that the correlation time of the laserinduced speckles was generally of the order of $10 \mathrm{~ms}$, and the experimental images with the exposure time of $1.7 \mathrm{~s}$ have the speckle effect averaged out. Therefore the coherence effect was not important in our images of Figs. 4 and 7.

Theory and experiment were also compared on the much studied cloverlike cross-polarized backscattering patterns for various concentrations of spherical scatterers and different particle sizes. Good quan- 
titative agreement was obtained. It was shown that these cloverlike patterns should emerge under the same general conditions described above. However, if particle concentration increases, the pattern shrinks around the point of incidence of the laser beam and eventually disappears.

This research was partially supported by the Office of Naval Research under contract N00014-95-1-0275, the Office of Vice President for Research at Texas A\&M University, and the Whitaker Foundation.

\section{References}

1. A. H. Hielscher, J. R. Mourant, and I. J. Bigio, "Influence of particle size and concentration on the diffuse backscattering of polarized light from tissue phantoms and biological cell suspensions," Appl. Opt. 36, 125-135 (1997).

2. A. I. Carswell and S. R. Pal, "Polarization anisotropy in lidar multiple scattering from clouds," Appl. Opt. 19, 4123-4126 (1980).

3. S. R. Pal and A. I. Carswell, "Polarization anisotropy in lidar multiple scattering from atmospheric clouds," Appl. Opt. 24, 3464-3471 (1985).

4. S. L. Jacques, M. Ostemeyer, L. Wang, and D. Stephens, "Polarized light transmission through skin using video reflectometry: toward optical tomography of superficial tissue layers," in Lasers in Surgery: Advanced Characterization, Therapeutics, and Systems VI, R. R. Anderson, ed., Proc. SPIE 2671, 199-220 (1996).

5. S. G. Demos and R. R. Alfano, "Optical polarization imaging," Appl. Opt. 36, 150-155 (1997).

6. A. H. Hielscher, A. A. Eick, J. R. Mourant, and I. J. Bigio, "Biomedical diagnostic with diffusely backscattered linearly and circularly polarized light," in Biomedical Sensing Imaging and Tracking Technologies II, T. Va-Dinh, R. A Lieberman, and G. G. Vuvek, eds. Proc. SPIE 2976, 298-305 (1997).

7. M. Dogariu and T. Asakura, "Photon pathlength distribution from polarized backscattering in random media," Opt. Eng. 35, 2234-2239 (1996).

8. A. H. Hielscher, A. A. Eick, J. R. Mourant, D. Shen, J. P. Freyer, and I. J. Bigio, "Diffuse backscattering Mueller matrices of highly scattering media," Opt. Exp. 1, 441-454 (1997).

9. W. S. Bickel and W. M. Bailey, "Stokes vectors, Mueller matrices, and polarized scattered light," Am. J. Phys. 53, 468-478 (1985).

10. A. Ambirajan and D. C. Look, "A backward Monte Carlo study of the multiple scattering of a polarized laser beam," J. Quantum Spectrosc. Radiat. Transfer 58, 171-192 (1997).

11. M. J. Raković and G. W. Kattawar, "Theoretical analysis of polarization patterns from incoherent backscattering of light," Appl. Opt. 37, 3333-3338 (1998).

12. B. D. Cameron, M. J. Raković, M. Mehrubeoglu, G. Kattawar, S. Rastegar, L. V. Wang, and G. Coté, "Measurement and calculation of the two-dimensional backscattering Mueller matrix of a turbid medium," Opt. Lett. 23, 485-487 (1998); erratum, 23, 1630 (1998).

13. H. C. van de Hulst, Light Scattering by Small Particles (Dover, New York, 1981).

14. S. Chandrasekhar, Radiative Transfer (Oxford U. Press, London, 1950).

15. G. Bohren and D. Hoffman, Absorption and Scattering of Light by Small Particles (Wiley, New York, 1983).

16. J. W. Hovenier and J. F. de Haan, "Polarized light in planetary atmospheres for perpendicular directions," Astron. Astrophys. 146, 185-191 (1985). 